\title{
Overview of Systematic Reviews and Meta-Analyses of Acupuncture for Stroke
}

\author{
Jun-huai Zhang Deren Wang Ming Liu \\ Stroke Clinical Research Unit, Department of Neurology, West China Hospital, Sichuan University, \\ Chengdu, PR China
}

\section{Key Words}

Overview · Systematic review · Meta-analysis .

Complementary therapy $\cdot$ Acupuncture $\cdot$ Stroke

\begin{abstract}
Background: We aimed to systematically overview published systematic reviews and meta-analyses in order to identify whether and when acupuncture is an effective treatment for stroke and stroke-related disorders. We also hoped to identify the best directions for future research in this area. Methods: Systematic reviews and meta-analyses of randomized controlled trials (RCTs) and quasi-RCTs evaluating the efficacy of acupuncture to treat stroke or stroke-related conditions were included. Electronic searches were conducted in the Cochrane Database of Systematic Reviews, Ovid MEDLINE, CINAHL, Ovid EMBASE, EBSCO Allied and Complementary Medicine (AMED) database, Chinese Biological Medicine Database, and Chinese National Knowledge Infrastructure Database. Two authors independently assessed the compliance of studies with eligibility criteria, and extracted data from included studies. The quality of systematic reviews was assessed according to the Overview Quality Assessment Questionnaire. Results: A total of 24 systematic reviews were included, of which 4 (16.7\%) were Cochrane systematic reviews and 20 (83.3\%) were non-Cochrane reviews. Acupuncture was analyzed as an acute stroke intervention in 3 re-
\end{abstract}

views (12.5\%), as an approach to stroke rehabilitation in 6 $(25 \%)$, and as an intervention to treat various stroke-related disorders in the remaining 15 (62.5\%). Reviews analyzing death or dependency/disability as the primary outcome reported no statistically significant difference between acupuncture and nonacupuncture control treatments. In contrast, reviews in which the outcome was improvement in global neurological deficit scores or performance on the video-fluoroscopic swallowing study test or water-swallowing test often reported that acupuncture was superior to control treatment. The quality of 10 reviews was 'poor', 6 reviews were 'moderate' and 8 were 'good'. Conclusions: The available evidence suggests that acupuncture may be effective for treating poststroke neurological impairment and dysfunction such as dysphagia, although these reported benefits should be verified in large, well-controlled studies. On the other hand, the available evidence does not clearly indicate that acupuncture can help prevent poststroke death or disability, or ameliorate other aspects of stroke recovery, such as poststroke motor dysfunction. These findings suggest that researchers should focus on the potential application of acupuncture to treat poststroke neurological impairment and dysfunction and on the development of more precise tools to assess these improvements after stroke.

\section{J.Z. and D.W. contributed equally to this study.}

\section{KARGER}

E-Mail karger@karger.com

www.karger.com/ned
(C) 2013 S. Karger AG, Base

0251-5350/14/0421-0050\$39.50/0
Prof. Ming Liu

Stroke Clinical Research Unit, Department of Neurology

West China Hospital, Sichuan University

No. 37 Guo Xue Xiang, Chengdu, Sichuan Province, 610041 (PR China)

E-Mail wyplmh@hotmail.com 


\section{Introduction}

Stroke is the second most common cause of death worldwide, as well as the leading cause of adult disability $[1,2]$. Nevertheless, medicine has developed few effective interventions against stroke and stroke-related disorders such as poststroke motor dysfunction and poststroke dysphagia. In China and elsewhere in East Asia, acupuncture has been a primary medical intervention for stroke and stroke-related disorders over the past 1,000 years [3], and in that part of the world, it continues to be used as a standard complementary therapy after stroke. In fact, a nationwide survey of 1,095 physicians from 247 Chinese hospitals between 1993 and 1994 found that $66 \%$ of Chinese doctors routinely used acupuncture to treat stroke patients, and $63 \%$ believed it to be effective [4]. Another more recent survey in 2007 among 202 Chinese acute stroke patients showed that $63.14 \%$ would choose acupuncture as part of medical intervention [5]. In South Korea an investigation of 304 stroke-patient respondents between 2004 and 2005 found that $54 \%$ had used complementary and alternative therapies, of which $92 \%$ had used traditional Korean medicine, primarily acupuncture [6]. Physicians and patients in Western countries and emerging economies [7-12] have also begun to accept acupuncture as an alternative therapy for a variety of neurological disorders.

Traditional Chinese medicine theory believes that the very core of disease onset is disruption of qi, the body's 'vital energies'. Qi flows through a network of channels, called 'meridians', running along the surface of the human body. Inserting needles at certain acupoints located along the meridians restores qi flow, thereby influencing progression of the disease [3]. In classical manual acupuncture, needles are inserted into acupoints to achieve a 'de qi' reaction, in which the patient perceives an ache or heaviness in the area surrounding the needle, while the acupuncturist performs a biomechanical movement (grasping the needle) that restores the qi flow at the site of insertion [13]. In addition to classical manual acupuncture, various modified acupuncture therapies have entered into widespread use, including scalp acupuncture [14], warm acupuncture, electro-acupuncture, auricular acupuncture [15] and 'xinnaokaiqiao' acupuncture [16].

The technical simplicity, noninvasiveness and lack of adverse effects of acupuncture compared to Western pharmacological and surgical approaches have made it an attractive treatment option in the West and East alike. Since the 1970s, hundreds of trials have been carried out to test the efficacy of acupuncture to treat stroke. These trials have been performed using widely differing methodologies and clinically diverse populations. To help identify real effects amid noise due to confounders, numerous systematic reviews and meta-analyses have been carried out to examine the effects of acupuncture to treat different types of stroke and stroke-related disorders at different stages of disease. These systematic reviews and meta-analyses have reported sometimes confusing and even conflicting results, which may help explain why acupuncture is still widely regarded as an 'alternative' therapy. In an effort to definitively identify under what conditions acupuncture can be an effective stroke treatment, we performed a systematic overview of systematic reviews and meta-analyses in the literature. Our secondary goal was to identify gaps and ambiguities in the literature as a guide for future research.

\section{Methods}

We included systematic reviews and meta-analyses of randomized controlled trials (RCTs) and quasi-RCTs that evaluated the efficacy of acupuncture to treat any type of stroke or stroke-related disorders, including poststroke dysphagia, depression, hiccups, urinary incontinence, hand-shoulder syndrome and shoulder pain. Systematic reviews and meta-analyses involving any acupuncture modality were included. Systematic reviews in which studies involved other alternative therapies or drug therapy were included if these same nonacupuncture therapies were given to both the intervention (acupuncture) arm and the control arm (no acupuncture). Reviews were excluded if they: (1) included controlled clinical trials; (2) compared the efficacy of different acupuncture therapies, such that there was no nonacupuncture control group, or (3) were nonsystematic narrative reviews or overviews.

Using our prespecified inclusion criteria, we comprehensively searched the following databases with no language restrictions: the Cochrane Library (Issue 5, 2013), Ovid MEDLINE (1966 to June 2013), Cumulative Index to Nursing and Allied Health Literature (CINAHL, 1982 to June 2013), Ovid EMBASE (1980 to June 2013), Allied and Complementary Medicine (AMED, 1985 to June 2013), Chinese Biological Medicine Database (CBM, 1981 to June 2013), and China National Knowledge Infrastructure (CNKI, 1996 to June 2013). The following search terms were used: 'stroke', 'cerebrovascular accident', 'isch(a)emic stroke', 'infarction', 'apoplexy', 'h(a)emorrhage', 'acupuncture', 'acupressure', 'auricular', 'systematic review' and 'meta-analysis'.

In addition to these automated searches in databases, we manually searched four Chinese journals relevant to acupuncture (from 1980 to June 2013): Acupuncture Research, Chinese Acupuncture and Moxibustion, Journal of Clinical Acupuncture and Moxibustion, and Shanghai Journal of Acupuncture and Moxibustion.

We identified eligible reviews using a two-step selection process. First, two investigators (J.Z. and D.W.) independently screened the titles and abstracts to exclude reviews that obviously did not meet the prespecified criteria. Second, the same investigators read the full text of the potentially eligible reviews and discarded those failing to meet the inclusion criteria. Disagreements were resolved by discussion and consensus. 
The same two authors extracted the following data from included reviews: countries of the included trials, number of trials included, patient demographic and clinical data, main outcomes and conclusions. The quality of the included reviews was assessed using the Overview Quality Assessment Questionnaires (OQAQ). This instrument evaluates the scientific quality of a systematic review based on the answers to nine questions, generating an overall quality score ranging from 1 to 7 [17]. A score of 6-7 indicates a review of 'good' quality, 3-5 indicates a review of 'moderate' quality, and $0-3$ a review of 'poor' quality.

Data from reviews were quoted in the form of standardized mean difference (SMD), weighted mean difference (WMD), odds ratio (OR) or relative risk (RR), depending on what the review authors reported. Whenever possible, meta-analysis results were also reported with $95 \%$ confidence intervals (CI).

\section{Results}

Initially, 10,739 potentially relevant records were identified through electronic and manual searches. After screening titles and abstracts, 10,702 records were excluded and the remaining 37 reviews were read in full. Finally, 24 systematic reviews were included in this overview: 4 (16.7\%) Cochrane systematic reviews and 20 (83.3\%) non-Cochrane systematic reviews (fig. 1).

Table 1 summarizes the key findings of all systematic reviews. Among the 24 reviews, 18 (75\%) were conducted in China, 4 (16.7\%) in Korea, 2 (8.3\%) in the UK, 1 (4.2\%) in Canada and 1 (4.2\%) in Sri Lanka. The 24 reviews included 366 RCTs and quasi-RCTs, with each systematic review comprising 3-72 RCTs. Three reviews (12.5\%) analyzed acupuncture as an acute stroke intervention [18-20], $6(25 \%)$ analyzed it as an approach to stroke rehabilitation [21-26], and the remaining $15(62.5 \%)$ analyzed it as an intervention to treat various stroke-related disorders. Of these 15 reviews, 4 focused on poststroke motor dysfunction [27-30], 4 on poststroke dysphagia [31-34], 2 on poststroke depression $[35,36]$, and 1 review on each of poststroke hiccup [37], poststroke urinary incontinence [38], shoulder-hand syndrome [29], shoulder pain [39] and apoplectic aphasia [40]. The OQAQ quality of 10 reviews was rated as 'good', 6 were rated as 'moderate' and 8 as 'poor'.

\section{Acupuncture as an Intervention to Treat Acute Stroke}

Three reviews [18-20], comprising 27 RCTs and quasi-RCTs, examined the efficacy of acupuncture therapy as an intervention for acute stroke. All 3 reviews conducted meta-analyses. The Cochrane systematic review by Zhang et al. [20] investigated both acute ischemic and hemorrhagic stroke, while the two non-Cochrane systematic reviews focused on acute ischemic stroke.

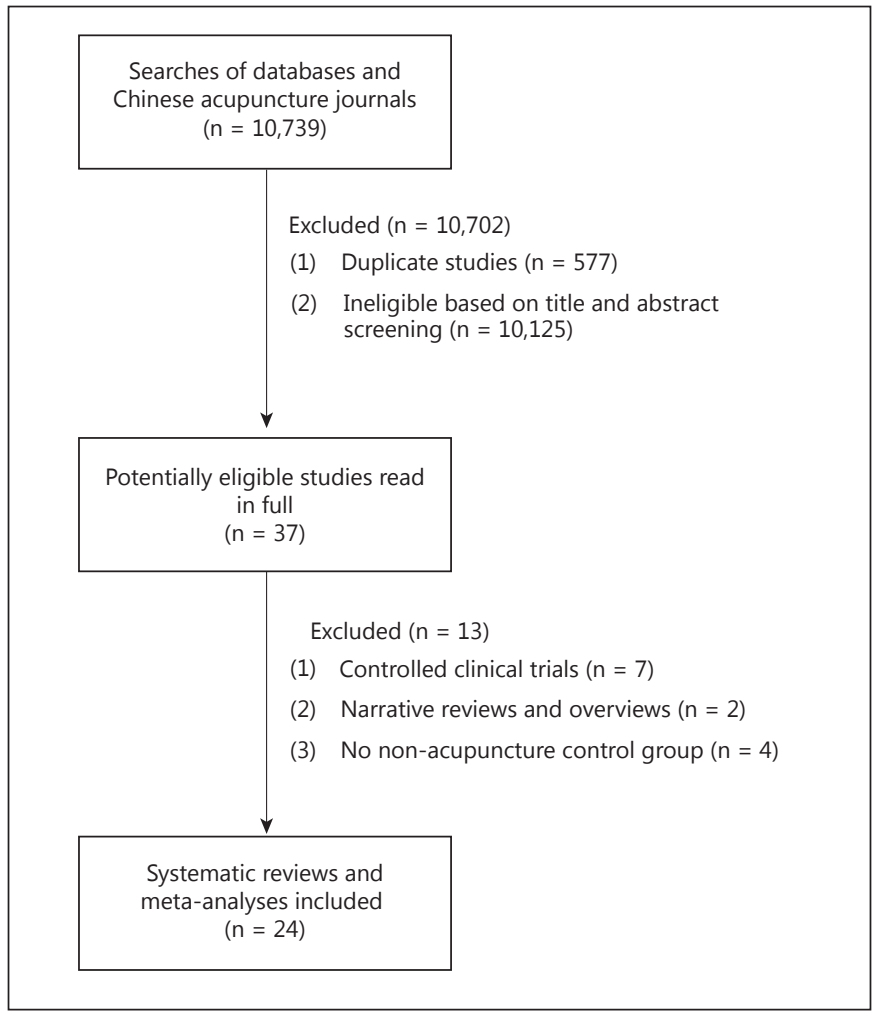

Fig. 1. Flowchart of the review selection process.

Two reviews assessed the efficacy of manual and electronic acupuncture against conventional care as the control [19], or against sham acupuncture or no treatment as the control [20]. The primary outcome in both reviews was death or dependency/disability at the end of treatment/follow-up, and in both cases there was no significant difference between the acupuncture and control arms. Nevertheless, Zhang et al. [20] discovered a borderline significant trend favoring acupuncture, with slightly lower rates of mortality and dependency at the end of long-term follow-up (15-22 months). The secondary outcome in both reviews was an improvement in neurological deficit scores. Both studies found acupuncture to be associated with significantly greater improvements than the control treatment: SMD 1.17, 95\% CI 0.30-2.04 [20] and WMD 3.49, 95\% CI 2.00-4.99 [19].

In the third review, Wang et al. [18] meta-analyzed 8 RCTs and compared the efficacy of scalp acupuncture with conventional medication. Acupuncture was associated with significantly greater improvement in neurological deficit scores (WMD 3.89, 95\% CI 2.43-5.36) as well as significantly higher rates of clinical efficacy (RR 1.23, 95\% CI 1.11-1.37).
Zhang/Wang/Liu 


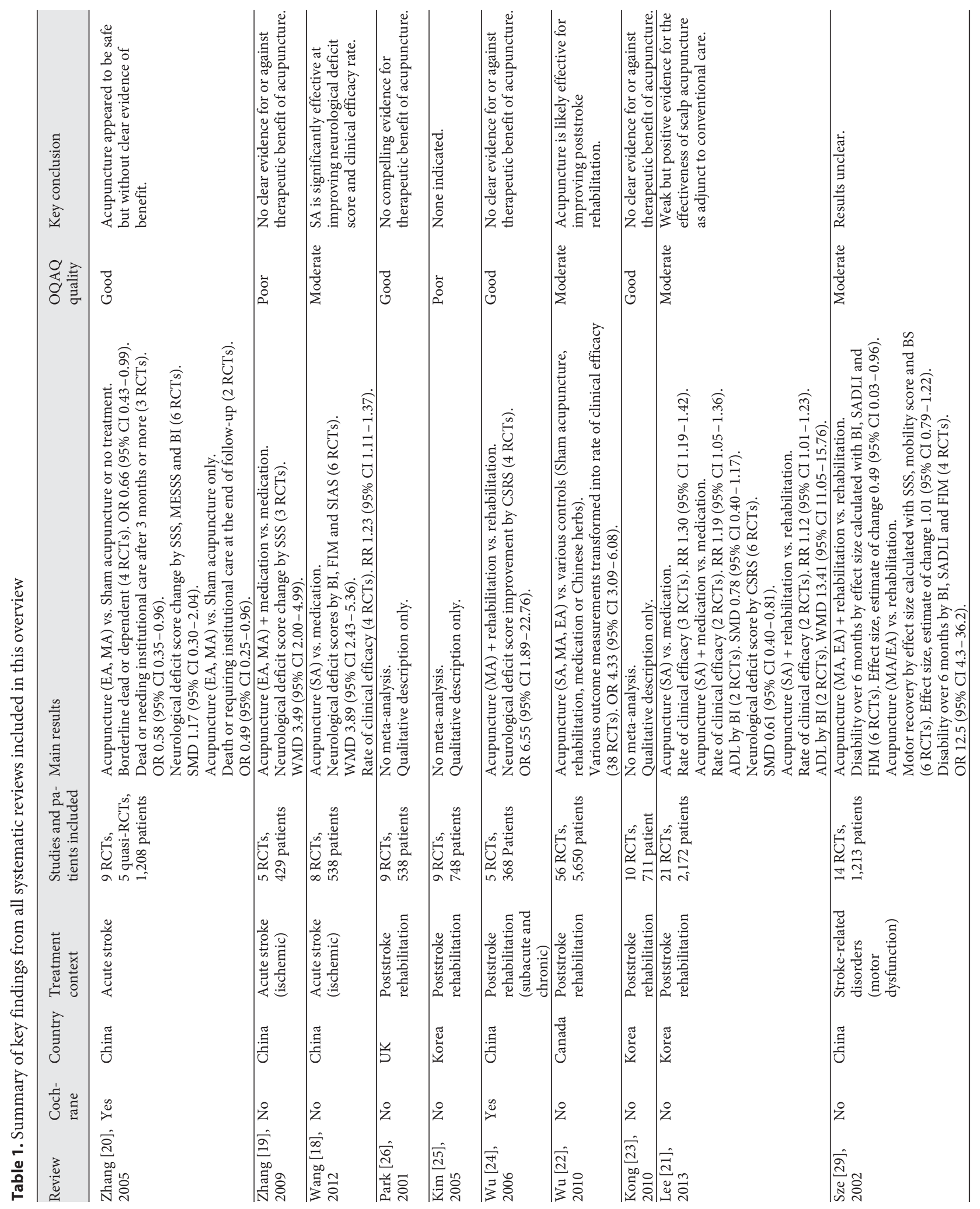




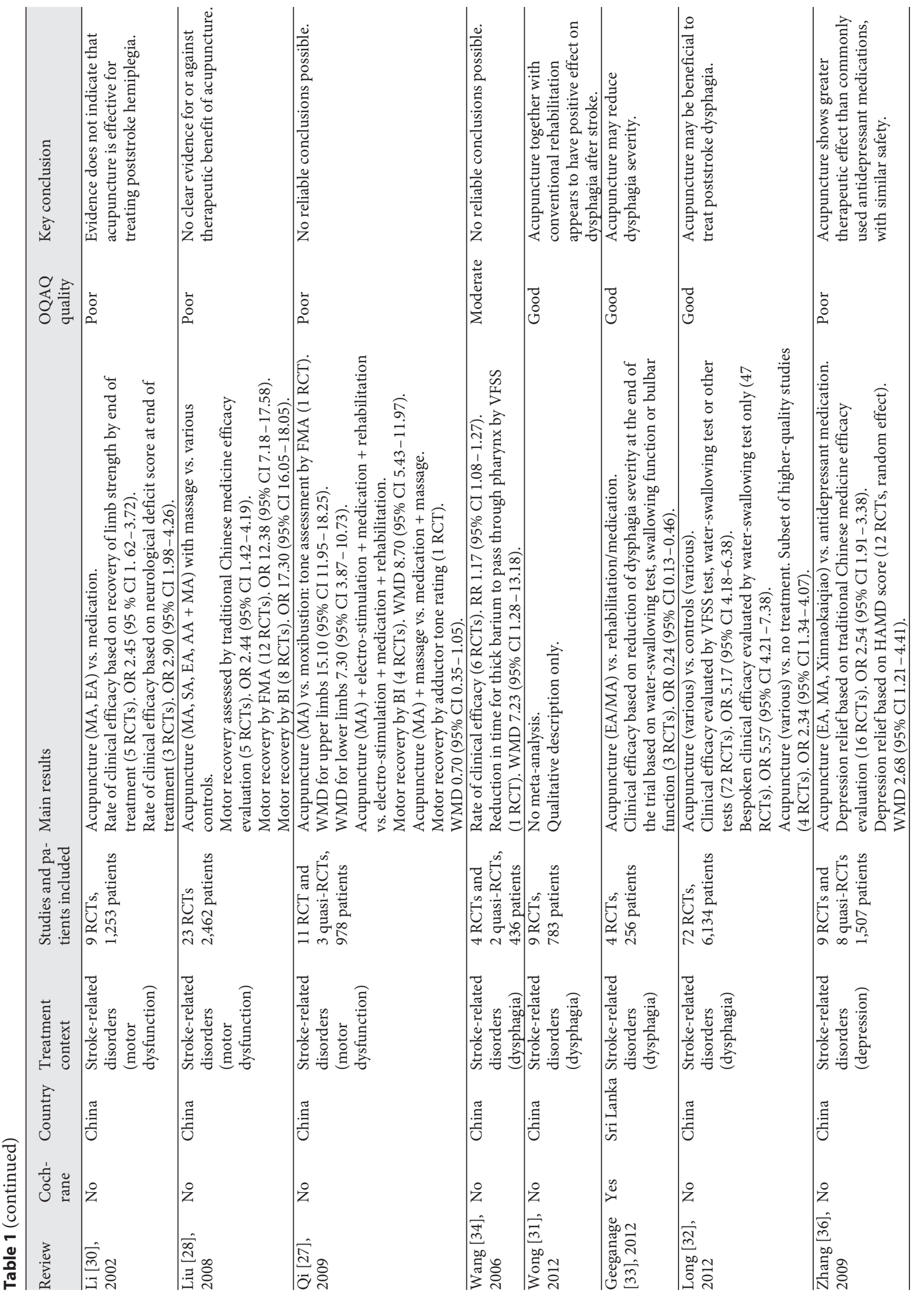




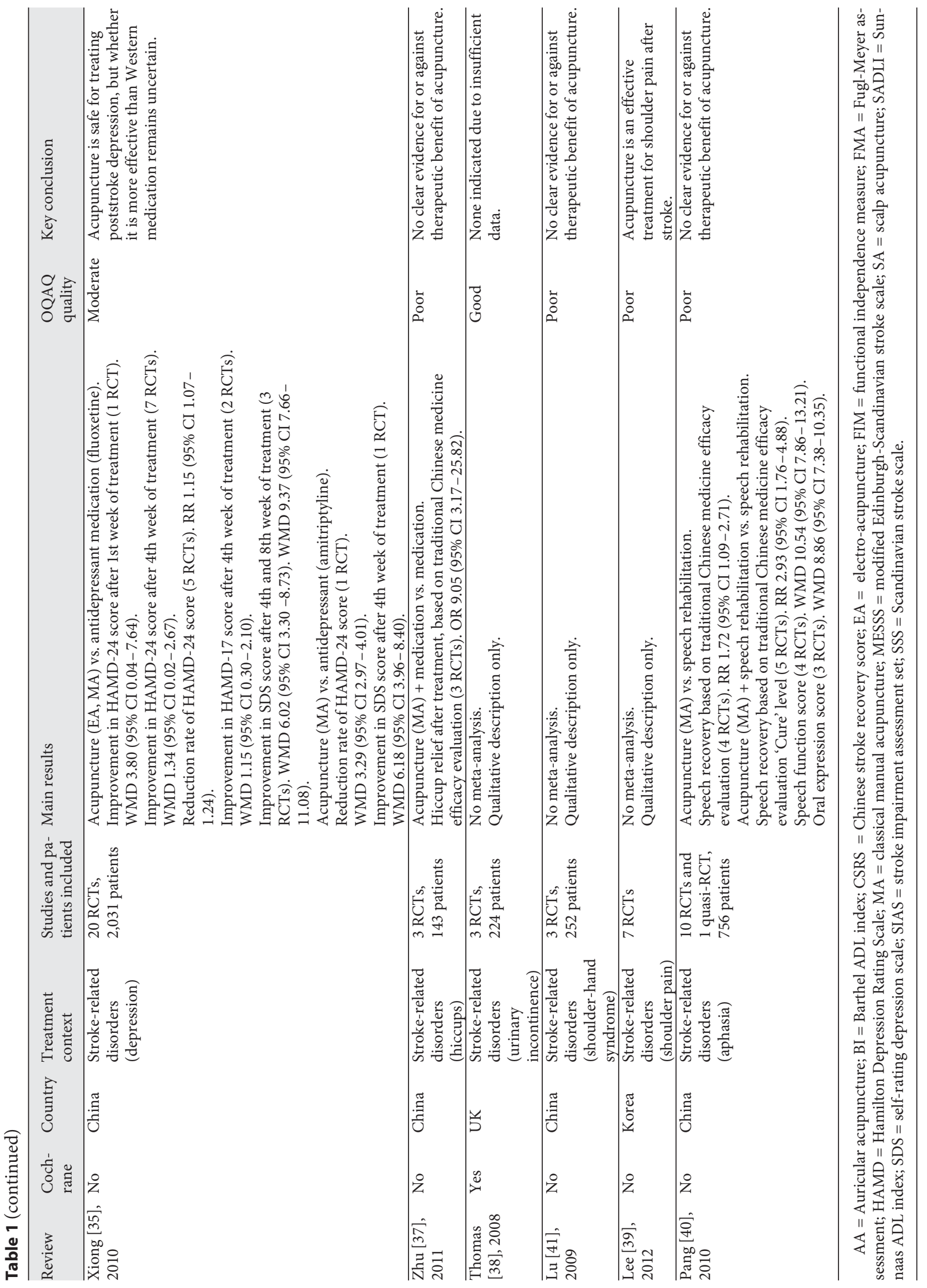




\section{Acupuncture for Poststroke Rehabilitation}

One Cochrane systematic review [24] and 5 nonCochrane systematic reviews $[21-23,25,26]$ assessed the efficacy of acupuncture for poststroke rehabilitation. All reviews focused on ischemic and hemorrhagic stroke, and the authors placed no restrictions on the disease stage. Five of these reviews assessed the efficacy of manual or electronic acupuncture against various control treatments including sham acupuncture, conventional rehabilitation, medication or Chinese herbs. Quantitative analyses were impossible because the included studies reported different outcomes, which spanned physical assessment, disability and motor assessments, and other validated stroke assessment scales. Nevertheless, three reviews tried to perform meta-analysis on subgroups to reduce the bias caused by heterogeneity [22-24]. The Cochrane review of Wu et al. [24] included subacute and chronic stroke patients and meta-analyzed changes in neurological deficit scores on the Chinese Stroke Recovery Scale. They found that acupuncture was associated with a much higher probability of a deficit reduction (OR 6.55, 95\% CI 1.89-22.76, $\mathrm{p}=$ $0.04)$, but the heterogeneity was high $\left(\mathrm{I}^{2}=68 \%\right)$. Kong et al. [23] meta-analyzed subgroups who received acupuncture or sham acupuncture, and found no significant difference in either functionality during activities of daily living (ADL) or in changes in neurological deficit scores. $\mathrm{Wu}$ et al. [22] transformed the diverse outcome measurements of 38 trials into binary variable outcomes and pooled the data. They found acupuncture to be associated with significantly better outcomes than sham or no acupuncture (OR 4.33, 95\% CI 3.09-6.08), leading them to conclude that acupuncture is likely to be effective for improving poststroke rehabilitation.

Among the 6 reviews assessing the efficacy of acupuncture for poststroke rehabilitation, only the review by Lee et al. [21] assessed the efficacy of scalp acupuncture. Those authors found that scalp acupuncture, when used as an adjunct to conventional treatment, was associated with higher rates of clinical efficacy and higher ADL functionality. Those authors concluded that the evidence for acupuncture as a useful part of poststroke rehabilitation was weak but positive.

\section{Acupuncture to Treat Stroke-Related Disorders}

Four reviews focused on poststroke motor dysfunction, and all of them performed meta-analyses. These reviews included studies on both hemorrhagic and ischemic stroke patients at different stages of disease [27-30]. Sze et al. [29] analyzed 14 RCTs that compared motor impairment and disability in patients treated with manu- al acupuncture alone, manual acupuncture with rehabilitation, or rehabilitation alone. A significant clinical benefit due to acupuncture was found only for severity of disability after treatment (OR 12.5, 95\% CI 4.3-36.2). Liu et al. [28] analyzed 23 RCTs comparing various acupuncture modalities (manual, electro, scalp, auricular) with various control treatments, which the review did not describe clearly. Acupuncture proved superior to control treatments for motor recovery, assessed using the Traditional Chinese Medicine Efficacy Evaluation (OR 2.44, 95\% CI 1.42-4.19) or the Fugl-Meyer Assessment (OR $12.38,95 \%$ CI 7.18-17.58), as well as for ADL functionality, measured using the Barthel Index (OR 17.30, 95\% CI 16.05-18.05). Li et al. [30] analyzed 9 RCTs that compared three acupuncture modalities (electro, scalp, manual) with conventional medication in terms of muscle tone improvement of hemiplegic limbs and neurological deficit scores at the end of treatment. Acupuncture was associated with significantly better muscle tone (OR 2.45, 95\% CI 1.62-3.72) and improvement in neurological deficit score (OR 2.90, 95\% CI 1.98-4.26). The fourth review, by Qi et al. [27], also found acupuncture to be associated with significantly greater improvement in ADL functionality, as measured by the Barthel Index (WMD 8.70, 95\% CI 5.43-11.97). However, none of the 4 reviews made firm conclusions about the efficacy of acupuncture due to the low quality of included trials or the small number of study participants.

One Cochrane review [33] and 3 non-Cochrane systematic reviews $[31,32,34]$ examined poststroke dysphagia. The Cochrane review by Geeganage et al. [33] compared the efficacy of deep-swallowing manual or electroacupuncture therapy with that of routine medication and rehabilitation for treating patients with acute or subacute stroke. Although the treatments did not differ significantly for the primary outcome of death or dependency/disability, acupuncture was associated with significantly less severe dysphagia (OR $0.24,95 \%$ CI $0.13-0.46$ ) based on a post-treatment water-swallowing test and bulbar function. Long and $\mathrm{Wu}$ [32] analyzed $72 \mathrm{RCT}$ s involving 6,134 patients with various types of stroke in which medicine and/or rehabilitation training plus acupuncture was compared with medicine and/or rehabilitation only using the video-fluoroscopic swallowing study (VFSS) test and the water-swallowing test as outcomes. Data for these outcomes were converted into clinical efficacy rates in order to allow quantitative synthesis of data from all trials. Meta-analysis showed that acupuncture was associated with significant improvement in the severity of dysphagia (OR 5.17, 95\% CI 4.18-6.38) when VFSS and water-swallow-
56

Neuroepidemiology 2014;42:50-58 DOI: $10.1159 / 000355435$
Zhang/Wang/Liu 
ing test results were combined. This clinical benefit was even greater when only water-swallowing test results were meta-analyzed (OR 5.57, 95\% CI 4.21-7.38). Long and $\mathrm{Wu}$ [32] verified this result in a subgroup analysis of 4 higher-quality RCTs (OR 2.34, 95\% CI 1.34-4.07). An earlier systematic review similarly reported that acupuncture was associated with significantly better VFSS and water-swallowing test results than were medication, rehabilitation training or Chinese herbs [34]. Wong et al. [31] made only qualitative comparisons in their review comparing the combination of acupuncture with swallowing training against either medication with training or medicine alone. Those authors concluded that acupuncture together with conventional rehabilitation might have a positive effect on dysphagia after stroke.

The remaining reviews included in our overview focused on other stroke-related disorders: 2 examined poststroke depression $[35,36]$ and single reviews examined poststroke hiccup [37], poststroke urinary incontinence [38], shoulder-hand syndrome [41], shoulder pain [39] and apoplectic aphasia [40]. Data in only one review on poststroke depression [36] and one on poststroke hiccup [37] suggested that acupuncture was superior to control treatments, but the authors in those studies did not draw any firm conclusions about the therapeutic efficacy of acupuncture due to the low quality of included trials or the small number of study participants.

\section{Discussion}

This overview identified a considerable number of systematic reviews, giving us a comprehensive picture of controlled assessments of acupuncture as a possible treatment for stroke and related disorders. Most studies focused on acupuncture as an intervention for stroke rehabilitation. Although most of the reviews we included did not wish to draw firm conclusions due to the small size of the included trials or their low methodological quality, our overview identified relatively convincing evidence for acupuncture as a treatment for stroke-related dysphagia.

Based on the ability of acupuncture to increase the flow of qi at points of needle insertion, practitioners of traditional Chinese medicine have historically held it to be particularly effective for ameliorating poststroke neurological impairment and dysfunction. Our overview supports this belief by providing evidence that acupuncture can be effective for stroke rehabilitation and strokerelated dysfunction based on assessments of neurological impairment, global neurological deficit, ADL functional- ity or water-swallowing ability. On the other hand, acupuncture was not associated with significantly better rates of death or dependency/disability than control treatments in any of the reviews we included.

It is worth noting that death or dependency/disability remain the primary outcomes accepted by most researchers and clinicians for assessing the efficacy of acupuncture. Other outcomes are less widely accepted because of potential measurement bias. For example, the clinical benefit of acupuncture for treating poststroke dysphagia may be partly attributable to the fact that the VFSS test and water-swallowing test are more subjective than measures of death and disability. Therefore, it would be valuable to develop more precise and objective tools that can be widely recognized for assessing the efficacy of acupuncture to treat stroke.

Our overview is constrained by limitations in the included systematic reviews. These limitations occur at the level of individual studies: only $13.5 \%$ of acupuncture trials included in Cochrane systematic reviews show acceptable quality in terms of randomization, allocation concealment, blinding and low rate of loss to follow-up [42]. In fact, many of the systematic reviews in our overview did not draw any firm conclusions because of methodological flaws in the included trials. In addition, many of the systematic reviews themselves failed to prespecify their inclusion and exclusion criteria according to the 'PICO' acronym. This may have led the authors to include inappropriate studies, causing significant heterogeneity in the dataset and increasing the risk of misleading conclusions according to the 'garbage in, garbage out' principle. One aspect of this heterogeneity is that many systematic reviews included different types of stroke (ischemic and hemorrhagic) at different stages.

In conclusion, the current evidence suggests that acupuncture is not effective for death or dependency/disability. However, acupuncture may be effective for treating poststroke neurological impairment and dysfunction, particularly poststroke dysphagia, although these findings require verification in rigorous RCTs. Future studies should place more emphasis on the efficacy of acupuncture for treating poststroke neurological impairment and dysfunction and on the development of more precise tools for assessing these outcomes.

\section{Disclosure Statement}

The authors have nothing to disclose. 


\section{References}

1 Murray CJ, Vos T, Lozano R, et al: Disabilityadjusted life years (DALYS) for 291 diseases and injuries in 21 regions, 1990-2010: a systematic analysis for the global burden of disease study 2010. Lancet 2012;380:2197-2223.

-2 Lozano R, Naghavi M, Foreman K, et al: Global and regional mortality from 235 causes of death for 20 age groups in 1990 and 2010: a systematic analysis for the global burden of disease study 2010. Lancet 2012;380:20952128.

3 Zhang S, Li N, Liu M: Use of acupuncture for stroke in China. Acupunct Med 2009;27:146.

-4 Liu M, Wu B, Wang WZ, Lee LM, Zhang SH, Kong LZ: Stroke in China: epidemiology, prevention, and management strategies. Lancet Neurol 2007;6:456-464.

5 Li N, Wang CW, Lv JQ: Survey study of conditions of patients with acute stroke seeking for acupuncture treatment (in Chinese). Zhongguo Zhen Jiu 2009;29:1009-1012.

6 Shin YI, Yang CY, Joo MC, Lee SG, Kim JH, Lee MS: Patterns of using complementary and alternative medicine by stroke patients at two university hospitals in Korea. Evid Based Complement Alternat Med 2008;5:231-235.

$>7$ Pandian JD, Liu M, Misbach J, Venketasubramanian N: Alternative therapies for stroke treatment in Asia. Int J Stroke 2011;6:541543.

$>8$ Shah SH, Engelhardt R, Ovbiagele B: Patterns of complementary and alternative medicine use among United States stroke survivors. J Neurol Sci 2008;271:180-185.

$>9$ Bell IR: Adjunctive care with nutritional, herbal, and homeopathic complementary and alternative medicine modalities in stroke treatment and rehabilitation. Top Stroke Rehabil 2007;14:30-39.

-10 Thomas KJ, Nicholl JP, Coleman P: Use and expenditure on complementary medicine in England: a population based survey. Complement Ther Med 2001;9:2-11.

$>11$ MacPherson H, Thomas K, Walters S, Fitter M: The York acupuncture safety study: prospective survey of 34,000 treatments by traditional acupuncturists. BMJ 2001;323:486487.

-12 Eisenberg DM, Davis RB, Ettner SL, Appel S, Wilkey S, Van Rompay M, Kessler RC: Trends in alternative medicine use in the United States, 1990-1997: results of a follow-up national survey. JAMA 1998;280:1569-1575.

$\checkmark 13$ Lundeberg T: To be or not to be: the needling sensation (de qi) in acupuncture. Acupunct Med 2013;31:129-131.
14 Kim MK, Choi TY, Lee MS, Lee H, Han CH: Contralateral acupuncture versus ipsilateral acupuncture in the rehabilitation of poststroke hemiplegic patients: a systematic review. BMC Complement Altern Med 2010;10: 41.

15 Nogier P: Face to face with auriculotherapy. Acupunct Electrother Res 1983;8:99-100.

16 Luo D, Fan XN, Shi XM: The Xingnao Kaiqiao acupuncture and 'the same treatment for different diseases'. J Clin Acu Moxibustion 2011; 2011:1-3.

17 Oxman AD, Guyatt GH: Validation of an index of the quality of review articles. J Clin Epidemiol 1991;44:1271-1278.

18 Wang Y, Shen J, Wang XM, Fu DL, Chen CY, Lu LY, Lu L, Xie CL, Fang JQ, Zheng GQ: Scalp acupuncture for acute ischemic stroke: a meta-analysis of randomized controlled trials. Evid Based Complement Alternat Med 2012;2012:480950.

19 Zhang T, Zhang L, Zhang HM, Li Q: Systematic review of acupuncture therapy for acute ischemic stroke. China J Tradit Chin Med Pharm 2009;24:101-104.

20 Zhang SH, Liu M, Asplund K, Li L: Acupuncture for acute stroke. Cochrane Database Syst Rev 2005:CD003317.

21 Lee SJ, Shin BC, Lee MS, Han CH, Kim JI: Scalp acupuncture for stroke recovery: a systematic review and meta-analysis of randomized controlled trials. Eur J Int Med 2013;5: 87-99.

22 Wu P, Mills E, Moher D, Seely D: Acupuncture in poststroke rehabilitation: a systematic review and meta-analysis of randomized trials. Stroke 2010;41:e171-e179.

23 Kong JC, Lee MS, Shin BC, Song YS, Ernst E: Acupuncture for functional recovery after stroke: a systematic review of sham-controlled randomized clinical trials. CMAJ 2010;182:1723-1729.

24 Wu HM, Tang JL, Lin XP, Lau J, Leung PC, Woo J, Li YP: Acupuncture for stroke rehabilitation. Cochrane Database Syst Rev 2006:CD004131.

25 Kim EJ, Lee JD, Kang SK: The study on the effectiveness of acupuncture in stroke rehabilitation. J Kor Acu Moxibustion Soc 2005; 22:211-221.

26 Park J, Hopwood V, White AR, Ernst E: Effectiveness of acupuncture for stroke: a systematic review. J Neurol 2001;248:558-563.

27 Qi YZ, Fu LX, Xiong J, Wang ZL, Mou J, Lu YM: Systematic evaluation of acupuncture for treatment of post-stroke spastic paralysis. Chin Acu Moxibustion 2009;29:683-688.

28 Liu ZD, Li HY, Song Y, Zhang BH, Guo JW, Cai YF, Huang Y, Pei J: Systematic review of RCTs on acupuncture for post-stroke motor dysfunction. Shanghai J Acu Moxibustion 2008;27:38-42.
29 Sze FK, Wong E, Or KK, Lau J, Woo J: Does acupuncture improve motor recovery after stroke? A meta-analysis of randomized controlled trials. Stroke 2002;33:2604-2619.

30 Li N, Feng B, Zou J, Liu Y: Meta-analysis of acupuncture for semiplegia caused by stroke. J Chengdu Univ TCM 2002;25:37-39.

31 Wong IS, Ng KF, Tsang HW: Acupuncture for dysphagia following stroke: a systematic review. Eur J Int Med 2012;4:e141-e150.

32 Long YB, Wu XP: A meta-analysis of the efficacy of acupuncture in treating dysphagia in patients with a stroke. Acupunct Med 2012; 30:291-297.

33 Geeganage C, Beavan J, Ellender S, Bath PM: Interventions for dysphagia and nutritional support in acute and subacute stroke. Cochrane Database Syst Rev 2012;10:CD000323.

34 Wang LP, Xie Y: Systematic evaluation on acupuncture and moxibustion for treatment of dysphagia after stroke. Chin Acu Moxibustion 2006;26:141-146.

35 Xiong J, Du YH, Liu JJ, Lin XM, Sun P, Xiao L, Gao X, Chen YW: Acupuncture versus western medicine for post stroke depression: a systematic review. J Evid Based Med 2010; 10:179-184.

36 Zhang JB, Ren L, Sun Y: Meta-analysis on acupuncture for treatment of depression in patients of poststroke. Chin Acu Moxibustion 2009;29:599-602.

37 Zhu LL, Wang WX, Guo XG: Acupuncture for hiccups after stroke: a systematic review. Chin J Evid Based Med 2011;11:325-328.

38 Thomas LH, Cross S, Barrett J, French B, Leathley M, Sutton CJ, Watkins C: Treatment of urinary incontinence after stroke in adults. Cochrane Database Syst Rev 2008:CD004462.

39 Lee JA, Park SW, Hwang PW, Lim SM, Kook S, Choi KI, Kang KS: Acupuncture for shoulder pain after stroke: a systematic review. J Altern Complement Med 2012;18:818-823.

40 Pang Y, Wu LB, Liu DH: Acupuncture therapy for apoplectic aphasia: a systematic review. Chin Acu Moxibustion 2010;30:612-616.

41 Lu YM, Fu LX, Mu J, Xu HJ, Qi YZ: Acupuncture for post stroke shoulder hand syndrome: a systematic review. Chin J Evid Based Med 2009;9:976-978.

42 Wang DR, Yang WM, Liu M: Acupuncture for neurological disorders in the Cochrane reviews characteristics of included reviews and studies. Neural Regen Res 2011;6:440-443. 